\title{
Fatores associados à atividade educativa em saúde bucal na assistência pré-natal
}

\author{
Factors associated with educational guidance regarding oral health \\ during prenatal care
}

Flávia Carneiro Bastos de Souza Schwab (https://orcid.org/0000-0001-5660-2260) ${ }^{1}$

Lorena Ferreira (https://orcid.org/0000-0001-5707-5677) ${ }^{2}$

Katrini Guidolini Martinelli (https://orcid.org/0000- 0003-0894-3241) ${ }^{2}$

Carolina Dutra Degli Esposti (https://orcid.org/0000-0001-8102-7771) ${ }^{2}$

Karina Tonini dos Santos Pacheco (https://orcid.org/0000-0002-4687-6062) ${ }^{3}$

Adauto Emmerich Oliveira (https://orcid.org/0000-0002-9679-8592) ${ }^{2}$

Edson Theodoro dos Santos Neto (https://orcid.org/0000-0002-7351-7719) ${ }^{2}$

${ }^{1}$ Prefeitura Municipal de Vitória. Av. Mal.

Mascarenhas de Moraes 1927, Bento Ferreira. 29050-945 Vitória ES Brasil.odontologa_ES@ hotmail.com

${ }^{2}$ Programa de Pós-

Graduação em Saúde

Coletiva, Universidade

Federal do Espírito Santo

(UFES). Vitória ES Brasil.

${ }^{3}$ Departamento de Medicina Social, UFES. Vitória ES

Brasil.

\begin{abstract}
The scope of this article is to verify the factors associated with providing educational guidance related to oral health during prenatal care in the Metropolitan Region of Greater Vitória - ES, Brazil. It involved a cross-sectional study of 1,035 postpartum women in public or affiliated private hospitals in the SUS in 2010. A descriptive analysis of the main educational information and binomial logistic regression was conducted to test the association between individual and contextual variables and "receiving educational guidance through meetings and/or pregnant mother groups in the health unit and/or through home visits". The information given most during prenatal care was "the advantage of exclusive breastfeeding until six months of life" (52.0\%) and least was "the importance of fluoride use in health" (18.9\%). Being attended by the Family Health Strategy (FHS) $(O R=1.94)$, having adequate living conditions for women and children $(O R=1.73)$ and an additional prenatal visit $(O R=1.08)$, increased the chance of providing educational guidance in prenatal care. Frequent pre-natal visits, coverage of health services in line with the FHS, and adequate conditions of housing were factors that were positively associated with educational guidance during prenatal care.
\end{abstract}

Key words Maternal and child health, Prenatal education, Unified Health System, Family Health
Resumo O objetivo deste artigo é verificar os fatores associados à realização de atividades educativas relacionadas à saúde bucal durante o acompanhamento pré-natal na Região Metropolitana da Grande Vitória-ES, Brasil. Estudo transversal com 1.035 puérperas em maternidades públicas ou conveniadas ao SUS, em 2010. Executou-se análise descritiva das principais informações educativas e regressão logística binomial associandose variáveis individuais e contextuais com o desfecho "recebimento de atividade educativa por meio de reuniões elou grupo de gestante na unidade de saúde elou por meio de visita domiciliar". A informação mais recebida durante o pré-natal foi: "vantagem da amamentação exclusiva até os seis meses de vida" (52,0\%) e a menos recebida foi " $a$ importância do uso do flúor na saúde" (18,9\%). Ter atendimento pela Estratégia Saúde da Família $(O R=1,94)$, ter condições externas de moradia adequadas $(O R=1,73)$ e aumento de 1 consulta pré-natal $(O R=1,08)$ aumentaram a chance de realização de atividade educativa no pré-natal. Número elevado de consultas pré-natal, cobertura dos serviços de saúde nos moldes da ESF e condições externas de moradia adequadas foram fatores que se associaram positivamente às atividades educativas durante o pré-natal.

Palavras-chave Saúde Materno-Infantil, Educação Pré-natal, Sistema Único de Saúde, Saúde da Família 


\section{Introdução}

A gravidez é caracterizada como um período de mudanças físicas e emocionais que motivam o acompanhamento pré-natal, com a prioridade do acolhimento à mulher e o oferecimento de respostas e de apoio aos seus sentimentos e dúvidas ${ }^{1}$. Esse acompanhamento tem como objetivo assegurar o adequado desenvolvimento da gestação, permitindo o parto de um recém-nascido saudável e segurança à saúde materna ${ }^{2}$.

Durante o período gestacional a mulher encontra-se mais receptiva e motivada em obter novos conhecimentos e absorver novas atitudes, modificando hábitos do seu dia a dia que possam influenciar a sua saúde e do feto em desenvolvimento. As gestantes fazem parte de um grupo estratégico para a educação em saúde bucal por incorporarem informações sobre saúde melhor do que em qualquer outro período de sua vida ${ }^{3}$. É importante que tal prática seja realizada multidisciplinarmente, seja de forma individual e/ou coletiva, por meio de grupos de gestantes, salas de espera, intervenções comunitárias e outros ${ }^{3}$.

Durante o acompanhamento pré-natal, fazse necessário o envolvimento da Equipe de Saúde Bucal (Cirurgião-Dentista, Técnicos em Saúde Bucal e Auxiliares em Saúde Bucal) juntamente com outros profissionais no processo de trabalho, para o desenvolvimento de atividades educativas e de apoio à gestante e seus familiares, tais como acompanhar o processo de aleitamento materno e os cuidados com o futuro recém-nascido, enfatizando a importância da amamentação para a dentição e o desenvolvimento dos aparelhos fonador, respiratório e digestivo da criança, orientar a mulher sobre hábitos alimentares saudáveis e de higiene bucal, orientar sobre a periodicidade das consultas odontológicas e os trimestres de gestação indicados para a realização de tratamento odontológico ${ }^{4}$. Esse envolvimento também tem como objetivo desmistificar as crenças populares e instaurar hábitos saudáveis de dieta e de higiene bucal ${ }^{5,6}$.

A realização de ações educativas durante o período gestacional é uma forma de promover vínculo, acolhimento, humanização do cuidado, de melhorar a comunicação entre gestantes e profissionais da saúde, de melhorar a adesão e assimilação de informações ${ }^{7,8}$, que objetivam a apropriação do conhecimento sobre fatores de risco e de proteção à saúde, incluindo o aleitamento materno e a saúde bucal ${ }^{4}$.

Entretanto, a educação em saúde ainda é pouco valorizada em alguns serviços de saúde, por falta de estrutura física para execução das atividades $^{9}$; por ainda existir predominância do modelo biologicista e/ou curativista, focado no atendimento clínico-obstétrico, no cumprimento de protocolos, rotinas e atendimentos individuais; por falta de capacitação dos profissionais, principalmente, no que diz respeito à promoção de saúde ${ }^{7,9}$.

Nesse sentido, o objetivo deste trabalho foi verificar os fatores associados à realização de atividades educativas relacionadas à saúde bucal durante o acompanhamento pré-natal na Região Metropolitana da Grande Vitória (RMGV), Espírito Santo (ES), Brasil.

\section{Métodos}

Dados provenientes de um estudo transversal realizado na RMGV-ES no período de abril a setembro de 2010, intitulado "Avaliação da qualidade na assistência pré-natal na Região Metropolitana da Grande Vitória: acesso e integração dos serviços de saúde" ${ }^{10}$, foram utilizados neste artigo. A amostra desse estudo foi composta por 1.035 puérperas que tiveram seus partos em maternidades públicas ou conveniadas ao SUS localizadas na RMGV-ES, por ocasião do parto. A amostra foi definida pela fórmula de tamanho amostral para estimar a proporção de gestantes que realizaram consulta odontológica no pré-natal, considerando a população de 17.980 nascidos vivos em 2007, de acordo com o Sistema de Informação de Nascidos Vivos (SINASC). A proporção esperada foi igual a 50\% para maximizar a amostra, a precisão desejada foi de $4 \%$, o efeito do desenho igual a 1,5 e o nível de significância de $5 \%$. Esses cálculos resultaram num tamanho amostral de 872 mulheres. Aumentou-se em cerca de $30 \%$ para considerar as possíveis perdas, recusas ou ausência do cartão de gestante no local do parto, o que resultou em 1.131 abordagens e 1.006 cartões avaliados. A amostra foi representativa de puérperas residentes nos sete municípios da microrregião, que obedeceu à estratificação de acordo com as seguintes proporções de nascimentos: Cariacica (22,6\%), Fundão $(1,0 \%)$, Guarapari (6,3\%), Serra (26,3\%), Viana (3,7\%), Vila Velha $(22,2 \%)$ e Vitória (17,9\%).

Foram selecionados e treinados sete entrevistadores que visitaram pelo menos uma vez na semana todas as oito maternidades incluídas no estudo, que assistem cerca de $80 \%$ dos partos da região. Para aprimoramento do formulário de pesquisa e treinamento dos entrevistadores 
foi executado estudo piloto. As puérperas foram selecionadas aleatoriamente, em todos os dias da semana, inclusive fins de semana e feriados. Os dados foram coletados por meio de entrevistas face a face com as puérperas, até 48 horas após o parto.

No dia da visita, todas as puérperas residentes em um dos municípios da RMGV-ES eram listadas em ordem aleatória, em seguida, realizavase a amostragem sistemática intercalando-se de duas em duas. Eram excluídas as mulheres que realizaram acompanhamento pré-natal (todo ou parte) no sistema privado; as que foram acompanhadas em municípios fora da RMGV-ES; as que fizeram pré-natal, mas não possuíam o "Cartão da Gestante" na hora da entrevista. Maiores detalhes sobre este estudo encontram-se em Santos Neto et al. ${ }^{10}$.

As variáveis do estudo foram construídas a partir de dados preenchidos nos questionários sobre atividades educativas relacionadas ao aleitamento materno e saúde bucal realizadas durante o pré-natal, que posteriormente foi relaciona$\mathrm{da}$ às características gestacionais, reprodutivas, demográficas e socioeconômicas.

Considerou-se que todas as informações recebidas dos profissionais de saúde pelas gestantes ocorreram por meio de atividades educativas durante o pré-natal, individuais ou coletivas, no momento da consulta individual, em reuniões de grupos ou em visitas domiciliares. No nível educativo investigou-se se algum profissional de saúde orientou ( $\operatorname{sim} /$ não) sobre: iniciar a amamentação dentro da primeira hora de vida; resolver problemas com relação à amamentação; posição da criança e da mãe para o aleitamento; como extrair o leite do peito; vantagem da amamentação exclusiva até os seis meses; continuar amamentando até os dois anos; amamentar sem horário marcado; como a criança deve sugar o peito para produzir leite; prejuízo do uso da mamadeira; prejuízo do uso da chupeta ou bico artificial; amamentar para o bom desenvolvimento dos ossos, músculos, dentes; amamentar para prevenir problemas respiratórios; importância da limpeza da boca da mãe; importância da limpeza da boca da criança; importância da alimentação saudável; uso do flúor; e alterações na gengiva devido à gravidez. Registrou-se também se a informação foi recebida pela puérpera durante atividade educativa individual e/ou coletiva, por meio de reunião ou visita domiciliar.

Para análise dos fatores associados à "a puérpera ter recebido atividade educativa por meio de reuniões e/ou grupo de gestante na unidade de saúde e/ou por meio de visita domiciliar” utilizou-se um modelo hierarquizado, dividido em três níveis.

O primeiro nível é composto pelas variáveis socioeconômicas: classificação da escolaridade (analfabeta/fundamental/médio/superior); classificação econômica geral, conforme o Critério de Classificação Econômica Brasil 2014 - Base LSE 2012, em classes D/E, C1/C2, A/B (http//:www. abep.org/new/critérioBrasil.aspx.pdf.2012); chefe da família (a própria mulher/o companheiro/ outros); se a mulher ganha dinheiro atualmente em trabalho (não/sim); além das condições de moradia interna, externa e gerais.

De acordo com as recomendações de Vettore et al. ${ }^{11}$, as condições internas de moradia são avaliadas e classificadas em inadequadas quando a aglomeração humana domiciliar for excessiva, ou seja, quando a razão de moradores por cômodo do domicílio, excluindo-se banheiros e cozinha, foi maior do que dois, ausência de piso revestindo o chão, paredes feitas de argila, palha, madeira, plástico ou metal ou ausência de descarga no banheiro. As condições externas de moradia foram classificadas em inadequadas quando o sistema de esgoto era ausente ou existia valão a céu aberto na rua do domicílio ${ }^{12}$. Foram classificadas como condições gerais de moradia inadequadas nos casos de condições de moradia internas ou externas inadequadas; altamente inadequadas, para a ocorrência de condições de moradia internas e externas inadequadas; e adequadas, na existência de condições de moradia internas e externas adequadas ${ }^{12}$.

As variáveis demográficas e reprodutivas maternas utilizadas no segundo nível foram: idade materna (10-19/20-34/35 ou mais anos), paridade (primípara/multípara), situação conjugal (vive com companheiro/tem companheiro, mas não vive com ele/não tem companheiro), hábitos comportamentais, como o uso de bebida alcoólica (não/sim), o fumo durante a gravidez (não/ sim), o uso de algum tipo de droga (não/sim), satisfação do pai com a gravidez (não/sim);

O terceiro nível englobou as variáveis gestacionais, tais como: o número de consultas prénatal (de forma contínua), modalidade do serviço de saúde em que a mulher realizou o pré-natal (Unidade Básica de Saúde [UBS]/Programa de Agentes Comunitários em Saúde [PACS]/Estratégia em Saúde da Família [ESF]), doenças ou intercorrências na gestação - hipertensão (não/ sim), glicemia elevada/diabetes (não/sim), infecção de urina (não/sim), anemia (não/sim), outras doenças (não/sim). A mulher também foi 
questionada se algum profissional de saúde considerou sua gravidez de risco (não/sim, médio risco/sim, alto risco/sim) e se a mesma tentou interromper a gravidez atual (não/sim).

Revisou-se e adequou-se o banco de dados previamente digitado no programa SPSS for windows 16.0 (SPSS Inc., Chicago, EUA), selecionando-se as variáveis de interesse. Em seguida, realizou-se a estatística descritiva para as variáveis relacionadas às informações recebidas durante o pré-natal, com cálculo de frequências absolutas e relativas e de seus respectivos intervalos de confiança (95\%). Já a estatística inferencial englobou os testes Qui-quadrado de Pearson e/ou Exato de Fischer, e o teste t-Student para testar a associação entre a variável educativa relacionada à reunião de grupo e/ou visita domiciliar com variáveis socioeconômicas, demográficas e reprodutivas maternas e gestacionais $(\mathrm{p}<0,05)$. Posteriormente, realizou-se o modelo hierarquizado de Regressão Logística Binomial, para verificar a influência desses fatores na chance da "a puérpera ter recebido atividade educativa por meio de reuniões e/ou grupo de gestante na unidade de saúde e/ou por meio de visita domiciliar".

Inicialmente fez-se a análise univariada, com todas as variáveis de cada nível hierárquico. As variáveis que apresentavam $\mathrm{p} \geq 0,20$ foram descartadas. Criou-se então um modelo com as variáveis do nível socioeconômico que apresentaram $\mathrm{p}<0,20$. Nesse bloco, foram mantidas as variáveis que apresentassem $\mathrm{p}<0,05$. Num segundo passo, foram acrescentadas as variáveis maternas do nível demográfico-reprodutivo que, na análise univariada, tinham $p<0,20$. Nesse momento, as variáveis do segundo nível que apresentaram $\mathrm{p} \geq 0,05$ foram retiradas do modelo. As variáveis socioeconômicas que haviam sido significantes na primeira etapa do modelo multivariado foram mantidas, independentemente do nível de significância apresentado após a introdução das variáveis demográfico-reprodutivas maternas. Assim criou-se um modelo com dois níveis.

Posteriormente foram introduzidas as variáveis do terceiro nível (gestacional) que, na análise univariada, tinham $\mathrm{p}<0,20$. Foram mantidas no modelo as variáveis desse terceiro nível, recém -introduzidas, que apresentaram $p<0,05$, sem retirar as variáveis dos níveis precedentes. Assim, resultou um modelo final com três níveis. A ordem de entrada das variáveis em cada etapa obedeceu à significância estatística notada na análise univariada.

A pesquisa foi submetida ao Comitê de Ética em Pesquisa do Centro de Ciências da Saúde da
Universidade Federal do Espírito Santo (UFES). O Termo de Consentimento Livre e Esclarecido foi assinado pelas participantes.

\section{Resultados}

No que concerne ao acompanhamento pré-natal, das 1.035 puérperas entrevistadas, $29(2,8 \%)$ declararam não terem realizado acompanhamento pré-natal, porém quatro delas disseram ter recebido algum tipo de informação sobre aleitamento materno, saúde bucal ou alimentação saudável durante o período gestacional por um profissional da saúde. Apenas 42,2\% $(n=427)$ das puérperas receberam de cinco a dez informações, conforme demonstrado na Tabela 1.

Dentre as informações mais frequentemente recebidas pelas puérperas encontram-se a vantagem da amamentação exclusiva no peito até os seis meses $(52,0 \%)$, a importância de uma alimentação saudável $(51,4 \%)$ e iniciar a amamentação no peito dentro da primeira hora de vida (49,0\%). Destacaram-se com menor frequência o recebimento de informação sobre o uso do flúor $(18,9 \%)$ e de informações por meio de reuniões de grupo e/ou visita domiciliar $(25,0 \%)$ (Tabela $1)$.

Na Tabela 2 estão apresentadas as associações entre o fato de a puérpera ter recebido ou não informações por meio de reunião de grupo e/ou visita domiciliar e as variáveis socioeconômicas, demográficas, reprodutivas maternas e gestacionais. O recebimento de informações por meio de reunião de grupo e/ou visita domiciliar mostrou associação estatisticamente significativa com o número crescente de consultas pré-natal $(\mathrm{p}<0,001)$, cobertura de $\operatorname{ESF}(\mathrm{p}=0,000)$ e condições externas de moradia adequadas $(\mathrm{p}=0,003)$.

Contudo, na Regressão Logística Binomial foram utilizadas as variáveis com nível de significância menor que $20 \%$ na análise univariada, sendo elas: número de consultas pré-natal $(\mathrm{p}<0,001)$, classe econômica $(\mathrm{p}=0,111)$, paridade $(\mathrm{p}=0,169)$, cobertura dos serviços de saúde $(\mathrm{p}=0,000)$, condições externas de moradia $(\mathrm{p}=0,003)$ e condições gerais de moradia $(\mathrm{p}=0,133)$.

No modelo final permaneceram as variáveis: "número de consultas pré-natal", "cobertura dos serviços de saúde" e "condições externas de moradia". Nesse modelo verificou-se que a cada consulta pré-natal frequentada pela puérpera, aumenta-se a chance de receber atividade educativa por meio de reuniões de grupo ou visitas domiciliares em $8 \%$. O mesmo ocorreu quando as 
Tabela 1. Frequência das informações recebidas durante o pré-natal na Região Metropolitana da Grande Vitória-ES, Brasil, 2010.

\begin{tabular}{|c|c|c|c|c|c|}
\hline \multirow[t]{2}{*}{ Variáveis educativas } & \multirow{2}{*}{$\begin{array}{c}\text { Total }{ }^{\star} \\
(\% \text { válido })\end{array}$} & \multirow{2}{*}{$\begin{array}{l}\text { Sim } \\
(\mathbf{n})\end{array}$} & \multirow[t]{2}{*}{$\%$} & \multicolumn{2}{|c|}{$\begin{array}{c}\text { Intervalo de } \\
\text { confiança }(95 \%)\end{array}$} \\
\hline & & & & Mínimo & Máximo \\
\hline \multicolumn{6}{|l|}{ Recebeu informações sobre... } \\
\hline Iniciar a amamentação dentro da $1^{\text {a }}$ hora de vida & 1009 & 494 & 49,0 & 46,0 & 52,0 \\
\hline Resolver problemas com relação à amamentação & 1009 & 394 & 39,0 & 36,0 & 42,0 \\
\hline Posição da criança e da mãe para o aleitamento & 1010 & 392 & 38,8 & 36,0 & 42,0 \\
\hline Como extrair o leite do peito & 1010 & 378 & 37,4 & 34,0 & 40,0 \\
\hline Vantagem da amamentação exclusiva até os 6 meses & 1010 & 525 & 52,0 & 49,0 & 55,0 \\
\hline Continuar amamentando até os 2 anos & 1010 & 412 & 40,8 & 38,0 & 44,0 \\
\hline Amamentar sem horário marcado & 1009 & 439 & 43,5 & 40,0 & 46,0 \\
\hline Sugar o peito para produzir leite & 1009 & 477 & 47,3 & 44,0 & 50,0 \\
\hline Prejuízo do uso da mamadeira & 1009 & 380 & 37,7 & 35,0 & 41,0 \\
\hline Prejuízo do uso da chupeta ou bico artificial & 1010 & 377 & 37,3 & 34,0 & 40,0 \\
\hline $\begin{array}{l}\text { Amamentar para o bom desenvolvimento dos ossos, } \\
\text { músculos, dentes }\end{array}$ & 1010 & 423 & 41,9 & 39,0 & 45,0 \\
\hline Amamentar para prevenir problemas respiratórios & 1010 & 402 & 39,8 & 37,0 & 43,0 \\
\hline Importância da limpeza da boca da mãe & 1010 & 377 & 37,3 & 34,0 & 40,0 \\
\hline Importância da limpeza da boca da criança & 1010 & 342 & 33,9 & 31,0 & 37,0 \\
\hline Importância da alimentação saudável & 1010 & 519 & 51,4 & 48,0 & 54,0 \\
\hline Uso do flúor & 1010 & 191 & 18,9 & 17,0 & 21,0 \\
\hline Alterações na gengiva devido a gravidez & 1010 & 298 & 29,5 & 27,0 & 32,0 \\
\hline $\begin{array}{l}\text { Atividades educativas por meio de reunião ou visita } \\
\text { domiciliar }\end{array}$ & 1011 & 253 & 25,0 & 22,0 & 28,0 \\
\hline Recebeu de 5 a 10 informações educativas & 1012 & 427 & 42,2 & 39,0 & 45,0 \\
\hline
\end{tabular}

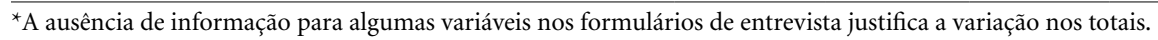

condições externas de moradia eram adequadas, aumentando cerca de $75 \%$ a chance de recebar a atividade educativa. Com relação à cobertura dos serviços de saúde, quando havia cobertura de ESF, a chance de realizar atividade educativa no pré-natal aumentou em cerca de $100 \%$ quando comparado ao PACS (Tabela 3).

\section{Discussão}

Nesta pesquisa, as mulheres que tiveram o acompanhamento pré-natal na RMGV-ES receberam em maior frequência atividades educativas relacionadas ao aleitamento materno, enquanto à saúde bucal foi abordada em menor frequência. Morar em um local minimamente urbanizado (com sistema de esgoto e sem valão a céu aberto), ter o acompanhamento pré-natal na ESF e ter um número crescente de consultas pré-natal mostrou associação com o recebimento de informações educativas por meio de reunião de grupo e/ou visita domiciliar.
Quando se considera o acompanhamento pré-natal, neste estudo, a maioria das mulheres declarou ter realizado mais de cinco consultas e somente $2,8 \%$ declararam não ter recebido acompanhamento pré-natal. Semelhantemente, outros autores observaram um número médio de consultas pré-natal de $7,5^{12}$ e $5,3^{13}$, com um percentual inferior a 5\% de mulheres que não realizaram acompanhamento pré-natal ${ }^{12,14}$. Isso indica que a alta cobertura de assistência pré-natal no Brasil $^{15}$ é uma realidade também na RMGV-ES.

Embora essa cobertura seja alta no Brasil e no Espírito Santo, quando se trata da realização de atividades educativas, ainda existem algumas dificuldades, visto que as informações recebidas pelas puérperas sobre aleitamento materno e saúde bucal alcançaram percentuais inferiores a $50 \%$. Deve-se reconhecer que dois aspectos podem estar envolvidos: ou a mulher recebeu as informações no pré-natal, sem que o processo educativo fosse efetivo, de modo a não conseguir retomar à memória as informações que se desejava construir, ou realmente as atividades educa- 
Tabela 2. Associação entre variável educativa relacionada a reunião de grupo e/ou visita domiciliar com variáveis independentes. Região Metropolitana da Grande Vitória-ES, Brasil, 2010.

\begin{tabular}{|c|c|c|c|c|c|c|}
\hline \multirow[t]{3}{*}{ Outras variáveis } & \multicolumn{6}{|c|}{$\begin{array}{l}\text { A puérpera recebeu atividade educativa por meio de } \\
\text { reuniões e/ou grupo de gestante na unidade de saúde } \\
\text { e/ou por meio de visita domiciliar? }\end{array}$} \\
\hline & \multicolumn{2}{|c|}{ Não } & \multicolumn{2}{|c|}{ Sim } & \multirow{2}{*}{$\chi^{2}$} & \multirow{2}{*}{ p-valor } \\
\hline & $\mathbf{n}$ & $\%$ & $\mathbf{n}$ & $\%$ & & \\
\hline \multicolumn{7}{|l|}{ Fatores socioeconômicos } \\
\hline \multicolumn{7}{|l|}{ Classificação da escolaridade } \\
\hline Analfabeta & 05 & 100 & 0 & 0 & & \\
\hline Fundamental & 367 & 75,5 & 119 & 24,5 & 2,00 & 0,571 \\
\hline Médio & 359 & 74,2 & 125 & 25,8 & & \\
\hline Superior & 21 & 72,4 & 08 & 27,6 & & \\
\hline \multicolumn{7}{|l|}{ Classificação econômica geral } \\
\hline $\mathrm{D} / \mathrm{E}$ & 212 & 78,8 & 57 & 21,2 & & \\
\hline $\mathrm{C} 1 / \mathrm{C} 2$ & 409 & 73,7 & 146 & 26,3 & 2,698 & 0,260 \\
\hline $\mathrm{A} / \mathrm{B}$ & 35 & 77,8 & 10 & 22,2 & & \\
\hline \multicolumn{7}{|l|}{ Quem é o chefe da família } \\
\hline A própria mulher & 82 & 74,5 & 28 & 25,5 & & \\
\hline O companheiro & 505 & 75,7 & 162 & 24,3 & 1,156 & 0,561 \\
\hline Outros & 163 & 72,1 & 63 & 27,9 & & \\
\hline \multicolumn{7}{|l|}{$\begin{array}{l}\text { Tem algum trabalho em que ganha dinheiro } \\
\text { atualmente }\end{array}$} \\
\hline Não & 540 & 75,1 & 179 & 24,9 & 0,004 & 0,950 \\
\hline Sim & 218 & 74,9 & 73 & 25,1 & & \\
\hline \multicolumn{7}{|l|}{ Condições internas de moradia } \\
\hline Inadequada & 118 & 75,6 & 38 & 24,4 & 0,065 & 0,798 \\
\hline Adequada & 631 & 74,7 & 214 & 25,3 & & \\
\hline \multicolumn{7}{|l|}{ Condições externas de moradia } \\
\hline Inadequada & 161 & 83,4 & 32 & 16,6 & 8,985 & 0,003 \\
\hline Adequada & 576 & 73,0 & 213 & 27,0 & & \\
\hline \multicolumn{7}{|l|}{ Condições gerais de moradia } \\
\hline Altamente inadequada & 39 & 84,8 & 07 & 15,2 & & \\
\hline Inadequada & 191 & 77,3 & 56 & 22,7 & 4,039 & 0,133 \\
\hline Adequada & 498 & 73,3 & 181 & 26,7 & & \\
\hline \multicolumn{7}{|l|}{ Fatores demográficos e reprodutivos } \\
\hline \multicolumn{7}{|l|}{ Idade } \\
\hline 10-19 anos & 168 & 75,3 & 55 & 24,7 & & \\
\hline 20-34 anos & 533 & 75,2 & 176 & 24,8 & 0,451 & 0,798 \\
\hline 35 ou mais anos & 56 & 71,8 & 22 & 28,2 & & \\
\hline \multicolumn{7}{|l|}{ Paridade } \\
\hline Primípara & 272 & 72,5 & 103 & 27,5 & 1,895 & 0,169 \\
\hline Multípara & 486 & 76,4 & 150 & 23,6 & & \\
\hline \multicolumn{7}{|l|}{ Situação conjugal } \\
\hline Vive com companheiro & 608 & 74,4 & 209 & 25,6 & & \\
\hline Tem companheiro, mas não vive com ele & 74 & 77,1 & 22 & 22,9 & 0,723 & 0,697 \\
\hline Não tem companheiro & 73 & 77,7 & 21 & 22,3 & & \\
\hline \multicolumn{7}{|l|}{ Uso de bebida alcoólica } \\
\hline Não & 686 & 74,6 & 234 & 25,4 & 0,816 & 0,366 \\
\hline Sim & 71 & 78,9 & 19 & 21,1 & & \\
\hline
\end{tabular}


Tabela 2. Associação entre variável educativa relacionada a reunião de grupo e/ou visita domiciliar com variáveis independentes. Região Metropolitana da Grande Vitória-ES, Brasil, 2010.

\begin{tabular}{|c|c|c|c|c|c|c|}
\hline \multirow[t]{3}{*}{ Outras variáveis } & \multicolumn{6}{|c|}{$\begin{array}{l}\text { A puérpera recebeu atividade educativa por meio de } \\
\text { reuniões e/ou grupo de gestante na unidade de saúde } \\
\text { e/ou por meio de visita domiciliar? }\end{array}$} \\
\hline & \multicolumn{2}{|c|}{ Não } & \multicolumn{2}{|c|}{ Sim } & \multirow{2}{*}{$\chi^{2}$} & \multirow{2}{*}{ p-valor } \\
\hline & $\mathbf{n}$ & $\%$ & $\mathbf{n}$ & $\%$ & & \\
\hline \multicolumn{7}{|c|}{ Você fumou durante a gravidez } \\
\hline Não & 667 & 74,9 & 224 & 25,1 & 0,053 & 0,817 \\
\hline Sim & 91 & 75,8 & 29 & 24,2 & & \\
\hline \multicolumn{7}{|c|}{ Usou algum tipo de droga } \\
\hline Não & 706 & 73,7 & 252 & 26,3 & - & - \\
\hline Sim & 14 & 100,0 & 0 & 0,0 & & \\
\hline \multicolumn{7}{|c|}{ O pai ficou satisfeito } \\
\hline Não & 68 & 75,6 & 22 & 24,4 & 0,030 & 0,863 \\
\hline Sim & 680 & 74,7 & 230 & 25,3 & & \\
\hline \multicolumn{7}{|c|}{ Fatores gestacionais } \\
\hline \multicolumn{7}{|c|}{ Cobertura dos serviços de saúde } \\
\hline UBS & 397 & 78,3 & 110 & 21,7 & & \\
\hline PACS & 114 & 82,6 & 24 & 17,4 & 18,883 & 0,000 \\
\hline ESF & 244 & 67,2 & 119 & 32,8 & & \\
\hline \multicolumn{7}{|l|}{ Glicemia/diabetes } \\
\hline Não & 530 & 74,6 & 180 & 25,4 & 0,561 & 0,454 \\
\hline Sim & 17 & 68,0 & 08 & 32,0 & & \\
\hline \multicolumn{7}{|l|}{ Hipertensão } \\
\hline Não & 694 & 75,0 & 231 & 25,0 & Fisher & 0,211 \\
\hline Sim & 08 & 100 & 0 & 0 & & \\
\hline \multicolumn{7}{|l|}{ Outras doenças } \\
\hline Não & 698 & 75,4 & 228 & 24,6 & 0,952 & 0,329 \\
\hline Sim & 60 & 70,6 & 25 & 29,4 & & \\
\hline \multicolumn{7}{|l|}{ Infecção de urina } \\
\hline Não & 414 & 76,0 & 131 & 24,0 & 0,108 & 0,742 \\
\hline Sim & 115 & 74,7 & 39 & 25,3 & & \\
\hline \multicolumn{7}{|l|}{ Anemia } \\
\hline Não & 401 & 73,2 & 147 & 26,8 & 1,220 & 0,269 \\
\hline Sim & 121 & 77,6 & 35 & 22,4 & & \\
\hline \multicolumn{7}{|c|}{ Gravidez considerada de risco } \\
\hline Não & 610 & 75,8 & 195 & 24,2 & & \\
\hline Sim, médio & 37 & 77,1 & 11 & 22,9 & 2,226 & 0,329 \\
\hline Sim, alto & 106 & 70,2 & 45 & 29,8 & & \\
\hline \multicolumn{7}{|c|}{ Tentou interromper essa gravidez } \\
\hline Sim & 720 & 75,1 & 239 & 24,9 & 0,064 & 0,800 \\
\hline Não & 36 & 73,5 & 13 & 26,5 & & \\
\hline
\end{tabular}

tivas não foram realizadas em momento algum, indicando que a assistência pré-natal se ateve às consultas médicas, mostrando o papel insuficiente do pré-natal na preparação das mulheres para a amamentação ${ }^{12}$, apesar deste ter sido o tema mais relatado pelas puérperas. Isso reflete dificuldades na implementação do programa de assistência pré-natal nos serviços de saúde, especialmente no que tange às atividades educativas.

Outros autores apontam que somente uma pequena proporção de mulheres $\left(27,5 \%{ }^{12}\right.$ e $36,9 \%{ }^{14}$ ) referiram ter participado de alguma atividade educativa durante o pré-natal, revelando uma falha da assistência, pois é durante esse 
Tabela 3. Regressão Logística Binomial entre variável educativa relacionada à reunião de grupo e/ou visita domiciliar e variáveis independentes. Região Metropolitana da Grande Vitória-ES, Brasil, 2010.

\begin{tabular}{|c|c|c|c|c|c|c|}
\hline \multirow{2}{*}{ Variáveis } & \multicolumn{3}{|c|}{ OR bruto } & \multicolumn{3}{|c|}{ OR ajustado } \\
\hline & OR & IC95\% & p-valor & OR & IC95\% & p-valor \\
\hline \multicolumn{7}{|c|}{$1^{\circ}$ Nivel - Variáveis socioeconômicas } \\
\hline \multicolumn{7}{|l|}{ Classificação econômica } \\
\hline $\mathrm{D} / \mathrm{E}$ & 1,00 & - & & 1,00 & - & \\
\hline $\mathrm{C}$ & 1,33 & $0,94-1,88$ & 0,111 & 1,27 & $0,88-1,83$ & 0,190 \\
\hline $\mathrm{A} / \mathrm{B}$ & 1,06 & $0,50-2,28$ & 0,876 & 1,02 & $0,47-2,21$ & 0,953 \\
\hline \multicolumn{7}{|c|}{ Condições externas de moradia } \\
\hline Inadequada & 1,00 & - & 0,008 & 1,00 & - & 0,048 \\
\hline Adequada & 1,86 & $1,23-2,80$ & & 1,94 & $1,01-3,73$ & \\
\hline \multicolumn{7}{|c|}{ Condições gerais de moradia } \\
\hline Altamente inadequada & 1,00 & - & & 1,00 & - & \\
\hline Inadequada & 1,63 & $0,69-3,85$ & 0,262 & 1,27 & $0,44-3,66$ & 0,656 \\
\hline Adequada & 2,03 & $0,89-4,61$ & 0,093 & 1,02 & $0,31-3,30$ & 0,978 \\
\hline \multicolumn{7}{|c|}{$1^{\circ}+2^{\circ}$ Níveis - Variáveis socioeconômicas + demográficas e reprodutivas maternas } \\
\hline \multicolumn{7}{|c|}{ Condições externas de moradia } \\
\hline Inadequada & 1,00 & - & 0,008 & 1,00 & - & 0,003 \\
\hline Adequada & 1,86 & $1,23-2,80$ & & 1,87 & $1,24-2,82$ & \\
\hline \multicolumn{7}{|l|}{ Paridade } \\
\hline Primípara & 1,23 & $0,92-1,64$ & 0,169 & 1,21 & $0,90-1,63$ & 0,210 \\
\hline Multípara & 1,00 & - & & 1,00 & - & \\
\hline \multicolumn{7}{|c|}{$1^{0}+2^{\circ}+3^{\circ}$ Níveis - Variáveis socioeconômicas + demográficas e reprodutivas maternas + gestacionais } \\
\hline \multicolumn{7}{|c|}{ Condições externas de moradia } \\
\hline Inadequada & 1,00 & - & 0,008 & 1,00 & - & 0,011 \\
\hline Adequada & 1,86 & $1,23-2,80$ & & 1,73 & $1,14-2,64$ & \\
\hline Número de consultas & 1,10 & $1,04-1,16$ & $<0,001$ & 1,08 & $1,02-1,14$ & 0,005 \\
\hline \multicolumn{7}{|c|}{ Cobertura dos serviços de saúde } \\
\hline PACS & 1,00 & - & & 1,00 & - & \\
\hline UBS & 1,32 & $0,81-2,14$ & 0,270 & 1,20 & $0,73-1,98$ & 0,464 \\
\hline ESF & 2,32 & $1,42-3,79$ & 0,001 & 1,94 & $1,17-3,20$ & 0,010 \\
\hline
\end{tabular}

Teste de Hosmer-Lemeshow do modelo final $=6,105 ; \mathrm{df}=8 ; \mathrm{p}=0,635$. Foi testada interação entre número de consultas pré-natal e cobertura dos serviços de saúde: p-valor=0,460.

momento que um espaço de educação em saúde deve ser criado, por meio da realização de práticas educativas voltadas para o desenvolvimento de capacidades individuais e coletivas, visando à melhoria da qualidade de vida e saúde ${ }^{16,17}$. Nesse sentido, os profissionais de saúde devem assumir postura de educadores que compartilham saberes no processo de trabalho, buscando devolver à mulher sua autoconfiança, considerando o prénatal e nascimento como momentos únicos para cada mulher ${ }^{18}$.

No entanto, a realidade dos serviços de saúde nem sempre responde às necessidades de saúde e expectativas sentidas pelas mulheres durante a gestação, pelo fato de, muitas vezes, não disporem de profissionais habilitados a realizar educa- ção em saúde no período gestacional e pelo modelo de assistência à saúde ainda estar bastante atrelado à prevenção de doenças ${ }^{19}$, excluindo-se as práticas de promoção da saúde.

Evidentemente, quando uma equipe de saúde não está sensibilizada para a importância da criação do vínculo com a gestante, aumenta-se o risco de desistência ou de menor frequência no acompanhamento pré-natal e nas ações de educação em saúde ${ }^{20,21}$, mesmo o Ministério da Saúde do Brasil salientando que ações educativas, vinculadas ao pré-natal são fundamentais para a gestante adquirir conhecimentos que visem uma melhor compreensão do processo gestacional ${ }^{4}$.

Dentre os temas mais abordados nas atividades educativas deste estudo e nas pesquisas de 
Carvalho e Araújo ${ }^{14}$ e Maeda et al. ${ }^{22}$, encontra-se o aleitamento materno, mostrando que as atividades educativas realizadas na assistência prénatal permanecem com uma postura tradicionalista. Nota-se a partir desses estudos ${ }^{14,22}$, que as informações comuns a outros campos de atuação profissional e disciplinar obtêm maior frequência de relato pelas puérperas. $\mathrm{O}$ recomendável seria que todas as gestantes fossem orientadas quanto à amamentação, visto que desde a década de 1980 há um esforço mundial por parte da Organização Mundial de Saúde, juntamente com o Fundo das Nações Unidas para a Infância, para promover e apoiar o aleitamento materno ${ }^{23}$.

Um estudo realizado no município de Vitória-ES, que integra a microrregião em estudo, seguiu uma coorte de recém-nascidos até completarem três anos. Os pesquisadores encontraram prevalência de aleitamento materno exclusivo até os seis meses de apenas $39,5 \%{ }^{24}$ e que o uso da chupeta e a introdução de outros bicos artificiais até o primeiro mês de vida são decisivos para aumentar o risco de interrupção do aleitamento materno ${ }^{25}$. Assim, é preciso refletir se a gestação está sendo conduzida de modo intervencionista, tornando a assistência e as atividades educativas fragmentadas, sem que a realidade da mulher gestante seja tratada na sua integralidade ${ }^{26}$, necessitando de outras ações para melhores resultados.

Este estudo mostrou que, além da insuficiência de informações sobre aleitamento materno exclusivo até os seis meses (frequências em torno de 50\%), as informações sobre saúde bucal obtiveram resultados ainda mais insatisfatórios, com frequências entre $20 \%$ e $40 \%$. No estudo de Garbin et al. ${ }^{27}, 80 \%$ das gestantes entrevistadas não receberam informações sobre saúde bucal durante a gestação. Situação semelhante foi revelada na pesquisa realizada com 121 puérperas internadas pelo SUS no Hospital Santa Helena no município de Cuiabá, Mato Grosso, Brasil, onde uma baixa proporção de gestantes recebeu orientação sobre cuidados relativos à sua própria saúde bucal $(24,7 \%)^{28}$.

Isso demonstra que informações sobre saúde bucal e cuidados de higiene bucal são pouco abordados durante o pré-natal, ou porque a equipe encontra-se carente de profissionais na área de saúde bucal para influenciar e contribuir nas ações educativas ou esses profissionais não estão adequadamente preparados para prestar assistência às gestantes no que diz respeito à promoção e prevenção em saúde bucal ${ }^{29}$, entendendo que as gestantes compõem um grupo priori- tário, com especificidades, medos e necessidades que merecem maior atenção.

A oportunidade de trocar informações sobre saúde bucal com a gestante durante o pré-natal deve ser bem aproveitada, pois a maioria das doenças bucais na gravidez pode ser prevenida ou amenizada com a instituição de um programa rigoroso de educação em saúde, com ênfase na promoção de saúde, que só terá êxito se contar com a colaboração e a necessária motivação da paciente para realizar mudanças de comportamento incentivadas pelo profissional de saúde ${ }^{3}$.

Contudo, deve-se ressaltar que pode haver outros fatores que favoreçam ou dificultem a participação das gestantes em atividades educativas de reuniões de grupo ou visita domiciliar.

Baseados nessa premissa, o presente estudo, ao testar a associação de diferentes variáveis socioeconômicas, demográficas, reprodutivas materna e gestacionais com o fato da puérpera ter recebido ou não informação educativa, observou que a realização de um número crescente de consultas pré-natal aumentava a chance de participação em atividades educativas durante o acompanhamento pré-natal, seja esta individual e/ou em grupo na unidade de saúde e/ou na visita domiciliar. O número de consultas pré-natal e o intervalo de tempo entre elas têm sido bastante discutidos na literatura científica, o que fomenta uma reflexão sobre o número ideal de consultas que assegure uma assistência de qualidade, que não comprometa a saúde materna e do embrião/ feto e que gere maior satisfação para a gestante $e^{30}$.

Entretanto, o número de consultas pré-natal e o momento de seu início não são suficientes para garantir a qualidade do pré-natal ${ }^{31}$, mas ter um número de consultas mínimo proporciona maior oportunidade de contato com o serviço e consequentemente maior chance de receber informações por meio de atividades educativas. No estudo realizado por Santos Neto et al. ${ }^{10}$, a alta prevalência de realização de consultas pré-natal e a quantidade total de consultas no primeiro e no terceiro trimestre foram fundamentais para condicionar uma assistência odontológica adequada. Dessa forma, o entrelaçamento entre as práticas profissionais na atenção primária torna-se fundamental para a produção de um cuidado prénatal qualificado e integral.

No que tange à cobertura de serviço, o acompanhamento pré-natal realizado em áreas de cobertura da ESF aumentou em cerca de 100\% a chance de realização de atividades educativas pelas gestantes neste estudo. No estudo de Anversa et al. ${ }^{31}$, as gestantes atendidas pela ESF realizaram 
mais frequentemente um pré-natal adequado em todos os níveis avaliados, receberam mais orientações durante a gestação e foram mais examinadas fisicamente, o que reforça a importância da ESF como modelo mais efetivo de organização da atenção, proporcionando o fortalecimento para reorganização da atenção primária à saúde no Brasil.

Isso reforça a resolutividade da ESF frente a outros modelos de atenção primária, destacando-se a organização do processo de trabalho e produção do cuidado, que estão diretamente ligadas à concepção de trabalho e à subjetividade de cada profissional e de cada usuário ${ }^{32}$. Na ESF, há um vínculo maior entre as equipes de saúde e as gestantes e seus familiares, que contribui para melhorar o acesso ao sistema de saúde pública e qualidade da atenção, resultando em maior satisfação do usuário quando comparados aos postos e centros de saúde tradicionais ou até mesmo algumas unidades de cuidado de saúde do setor privado ${ }^{33}$. Além disso, no território da ESF as orientações às comunidades e famílias são alcançadas por meio de visitas domiciliares dos agentes comunitários de saúde e do trabalho em escolas e organizações comunitárias ${ }^{33}$.

No entanto, fatores externos aos serviços de saúde também estão envolvidos no processo educativo das gestantes. Dentre estes, destaca-se como característica sociodemográfica, as condições externas de moradia, já que outras variáveis socioeconômicas foram exploradas nas análises iniciais sem apresentarem significância estatística. A falta de associação com escolaridade ou classe econômica pode ter ocorrido pelo fato das atividades educativas serem uma troca entre equipe de saúde e gestantes, e as equipes de saúde que já incorporaram as atividades educativas em sua rotina, convidarem todas as mulheres, sem exceção, a participar das atividades em grupo. Entretanto, as mulheres de baixa escolaridade ou renda, que são as de maior vulnerabilidade, deveriam receber maior atenção das equipes, talvez por meio de visitas domiciliares, pois são as que mais carecem de troca de informação, acolhimento e supervisão $0^{29}$.

Ademais, um estudo que avaliou a adequação da atenção pré-natal nas diferentes regiões brasileiras, de forma geral, encontrou que as áreas com melhores condições socioeconômicas, com melhores indicadores de renda e IDH, são as que têm maior frequência de adequação da $\operatorname{ESF}^{34}$, o que pode implicar na possibilidade dessas mulheres terem mais oportunidades de receberem atividades educativas no pré-natal.
Deve-se considerar, neste estudo, a presença de algumas limitações como a dificuldade na obtenção de respostas totalmente fidedignas concedidas pelas puérperas no pós-parto, visto que as mesmas poderiam responder positivamente sobre informações que receberam em acompanhamento pré-natal de filhos anteriores ou recebidas por meios de comunicação ou de experiências pessoais ou familiares, ou mesmo não se lembrarem com certeza dos fatos, denotando um possível viés de memória. Além disso, a associação deve ser interpretada com cautela, pois trata-se de um estudo transversal onde não há temporalidade, e a medida de associação (Odds Ratio) pode estar superestimada.

Sobre a realização de práticas educativas pelas gestantes durante o acompanhamento pré-natal, este estudo gera a reflexão da existência de fatores intervenientes, como a cobertura e o modelo de organização dos serviços de saúde, e as condições externas de moradia, que podem favorecer ou dificultar o acesso da gestante às informações qualificadas de saúde.

Nesse contexto a ESF destaca-se como o modelo de reorganização da atenção básica à saúde pública no Brasil, na qual as práticas educativas encontram condições mais favoráveis de modo a atingir o público-alvo das gestantes. Possibilita dessa forma, a incorporação de práticas saudáveis por toda a vida das mulheres, aumentando a corresponsabilização pela saúde, entre profissionais e usuárias, além de impulsionar a crescente autonomia quanto ao autocuidado e favorecer a qualidade da assistência pré-natal.

Contudo, há a necessidade de integração dos profissionais que compõem a equipe multiprofissional de atenção à gestante no pré-natal, com expansão da participação das equipes de saúde bucal. Essa ampliação tem o fim de promover integralidade na oferta de serviços de saúde pública, fornecendo respostas mais adequadas às necessidades de saúde das gestantes, levando-se em conta que as ações educativas são facilitadoras para o despertar de uma assistência pré-natal mais participativa e humanizada e que repercuta na qualidade de vida das usuárias, dos futuros recém-nascidos e suas famílias.

Tais resultados poderão subsidiar a reorientação ou adaptação das políticas de saúde, em especial as materno-infantis e de saúde bucal, e a forma como os serviços de saúde são organizados e ofertados, com ampliação do acesso às gestantes das atividades educativas e com o desenvolvimento de programas preventivo-educativos nos serviços de saúde. 


\section{Colaboradores}

FCBS Schwab: concepção, planejamento, análise, interpretação dos resultados e redação do trabalho. L Ferreira e KG Martinelli: análise, interpretação dos resultados, redação do trabalho e revisão final do trabalho. CDD Esposti e KTS Pacheco: interpretação dos resultados e revisão final do trabalho. AE Oliveira: planejamento, interpretação dos resultados e revisão final do trabalho. ET Santos Neto: concepção, planejamento, interpretação dos resultados e revisão final do trabalho. Todos os autores aprovaram a versão final encaminhada.

\section{Referências}

1. Brasil. Ministério da Saúde (MS). Secretaria de Políticos de Saúde. Área Técnica de Saúde da Mulher. Parto, aborto e puerpério: assistência humanizada à mulher. Brasília: MS; 2003.

2. Nunes JT, Gomes KRO, Rodrigues MTP, Mascarenhas MDM. Qualidade da assistência pré-natal no Brasil: revisão de artigos publicados de 2005 a 2015. Cad Saúde Colet 2016; 24(2):252-261.

3. Moimaz SAS, Zina LG, Serra FAP, Garbin CAS, Saliba NA. Análise da dieta e condição de saúde bucal em pacientes gestantes. Pesqui Bras Odontopediatria Clin Integr 2010; 10(3):357-363.

4. Brasil. Ministério da Saúde (MS). Secretaria de atenção à Saúde. Departamento de atenção básica. Atenção ao pré-natal de baixo risco. Brasília: MS; 2012.

5. Moreira PVL, Chaves AMB, Nóbrega MSG. Uma atuação multidisciplinar relacionada à promoção de saúde oral materno-infantil. Pesqui Bras Odontopediatria Clin Integr 2004; 4(3):259-264.

6. Braz G, Machado FC, Oliveira AS, Otenio CCM, Alves RT, Ribeiro RA. A experiência de um programa de atenção à saúde bucal no atendimento à gestante. $H U$ Rev 2010; 36(4):324-332.

7. Fogaça NR, Ferrari RAP, Gabani FL, Soares NTI. Operacionalização de grupos de pré-natal: percepção dos profissionais do serviço da atenção primária à saúde. Rev Pesqu Quali 2017; 5(7):128-142.

8. Guerra MI, Jucá V. Narratives of women on prenatal care in a public maternity. Psicol Saúde Doenças 2016; 17(2):253-264.

9. Bomfim ES, Slob EMGB, Oliveira BG, Ribeiro BS, Carmo ÉA, Santos PHS, Rosa RS. Práticas educativas do enfermeiro no cotidiano na estratégia de saúde da família. Rev Saúde Des 2016; 10(5):37-52.

10. Santos Neto ET, Oliveira AE, Zandonade E, Leal MC Acesso à assistência odontológica no acompanhamento pré-natal. Cien Saude Colet 2012; 17(11):30573068.

11. Vettore MV, Gama SGN, Schilithz AOC, Leal MC. Housing conditions as a social determinant of low birthweight and preterm low birthweight. Rev Saúde Pública 2010; 44(6):1021-1031.

12. Gonçalves MLC, Silva HO, Oliveira TA, Rodrigues LV, Campos ALA. Perfil sociodemográfico e obstétrico de puérperas assistidas no município de Iguatu-CE. Rev Bras Promoç Saúde 2012; 25(2 Supl.):33-39.

13. Trevisan MR, Lorenzi DRS, Araújo NM, Ésber K. Perfil da assistência pré-natal entre usuárias do Sistema Único de Saúde em Caxias do Sul. RBGO 2002; 24(5):293-299.

14. Carvalho VCP, Araújo TVB. Adequação da assistência pré-natal em gestantes atendidas em dois hospitais de referência para gravidez de alto risco do Sistema Único de Saúde, na cidade de Recife, Estado de Pernambuco. Rev Bras Saúde Matern Infant 2007; 7(3):309317.

15. Viellas EF, Domingues RMSM, Dias MAB, da Gama SGN, Theme Filha MM, Costa JV, Bastos MH, Leal MC. Assistência pré-natal no Brasil. Cad Saúde Pública 2014; 30(1):85-100.

16. Alvim DAB, Bassoto TRP, Marques GM. Sistematização a assistência de enfermagem à gestante de baixo risco. Rev Meio Amb Saúde 2007; 2(1):258-272. 
17. Pereira ALF. As tendências pedagógicas e a prática educativa nas ciências da saúde. Cad Saúde Pública 2003;19(5):1527-1534.

18. Rios CTF, Vieira NFC. Ações educativas no pré-natal: reflexão sobre a consulta de enfermagem como um espaço para educação em saúde. Cien Saude Colet 2007; 12(2):477-486.

19. Souza VB, Roecker S, Marcon SS. Ações educativas durante a assistência pré-natal: percepção de gestantes atendidas na rede básica de Maringá-PR. Rev Eletrônica Enferm 2011; 13(2):199-210.

20. Pozzer C, Silva AA, Ferreira CLL, Diaz C, Colomé J, Pereira ADA. Ações educativas em sala de espera com gestantes: uma alternativa para a promoção de saúde. Disciplinarum Scientia 2018; 19(2):207-224.

21. Figueiredo PP, Rossoni E. O acesso à assistência prénatal na atenção básica à saúde sob a ótica das gestantes. Rev Gaúch Enferm 2008; 29(2):238-245.

22. Maeda TC, Parreira BDM, Silva SR, Oliveira ACD. Importância atribuída por puérperas às atividades desenvolvidas no pré-natal. Rev Enferm Atenção Saúde 2014; 3(2):6-18.

23. World Health Organization (WHO). Department of nutrition for health and development. Department of child and adolescent health and development. The optimal duration of exclusive breastfeeding report of an expert consultation. Genebra: WHO; 2002.

24. Santos Neto ET, Emmerich AO, Zandonade E. O aleitamento materno exclusivo nos primeiros três meses de vida. Pediatria 2007; 29(2):89-98.

25. Santos Neto ET, Zandonade E, Emmerich AO. Analysis models for variables associated with breastfeeding duration. Rev Paulista Pediatr 2013; 31(3):306-314.

26. Delfino MRR, Patricio ZM, Martins AS, Silverio MR. $O$ processo de cuidar participante com um grupo de gestantes: repercussões na saúde integral individualcoletiva. Cien Saude Colet 2004; 9(4):1057-1066.

27. Garbin CAS, Sumida DH, Santos RR, Chehoud KA, Moimaz SAS. Saúde coletiva: promoção de saúde bucal na gravidez. Rev Odontol UNESP 2011; 40(4):161165.

28. Martins DP, Borges AH, Semenoff Segundo A, Palma VC, Volpato LER. A Saúde Bucal de uma Subpopulação de Gestantes Usuárias do Sistema Único de Saúde: um Estudo Piloto. Pesqui Bras Odontopediatria Clin Integr 2013; 13(3):273-278.
29. Ferreira SMSP, Silva JF, Silva RV, Pinheiro ÉS, Batista LD, Fernandes CG. Conhecimento em Saúde Bucal do Bebê e Expectativa Relativa ao Pré-Natal Odontológico: Retrato de Um Município Baiano de Grande Porte. Rev Faculdade Odontol Lins 2015; 25(2):19-30.

30. Pogliane RBS, Leal MC, Zandonade E, Santos Neto ET. Adequação do processo de assistência pré-natal segundo critérios do Programa de Humanização do Pré-natal e Nascimento e da Organização Mundial de Saúde. Cien Saude Colet 2014; 19(7):1999-2010.

31. Anversa ETR, Bastos GAN, Nunes LN, Pizzol TSD. Qualidade do processo da assistência pré-natal: unidades básicas de saúde e unidades de Estratégia Saúde da Família em município no Sul do Brasil. Cad Saúde Pública 2012; 28(4):789-800.

32. Bulgareli J, Cortellazzi KL, Ambrosano GMB, Meneghim MC, Faria ET, Mialhe FL, Pereira AC. A resolutividade em saúde bucal na atenção básica como instrumento para avaliação dos modelos de atenção. Cien Saude Colet 2014; 19(2):383-391.

33. Macinko J, Harris MJ. Brazil's Family Health Strategy - Delivering Community-Based Primary Care in a Universal Health System. N Engl J Med 2015; 372(23):2177-2181.

34. Guimarães WSG, Parente RCP, Guimarães TLF, Garnelo L. Acesso e qualidade da atenção pré-natal na Estratégia Saúde da Família: infraestrutura, cuidado e gestão. Cad Saúde Pública 2018; 34(5):e00110417.

Artigo apresentado em 27/11/2018

Aprovado em 20/05/2019

Versão final apresentada em 22/05/2019

Editores-chefes: Romeu Gomes, Antônio Augusto Moura da Silva 